\title{
VIBRATION FREQUENCIES OF A RECTANGULAR PLATE WITH LINEAR VARIATION IN THICKNESS AND CIRCULAR VARIATION IN POISSON'S RATIO
}

\author{
Amit Sharma \\ Amity University Haryana, Department of Mathematics, Gurugram, India \\ e-mail:dba.amitsharma@gmail.com
}

The frequency for the first two modes of vibration of a nonhomogeneous tapered rectangular plate is calculated under a bi linear (i.e., linear along both the axes) temperature field. For consideration of the nonhomogeneous material, the author assumed circular variation in Poisson's ratio. Tapering in the plate is assumed to be linear in one direction. The results are calculated for different values of plate parameters and presented with the help of graphs. Comparison of the results is also given, to support the results of the present study.

Keywords: rectangular plate, circular variation, linear variation, Poisson's ratio

\section{Symbols used}

$\begin{array}{ll}a, b & - \text { length and breadth of plate, respectively } \\ \zeta, \psi & - \text { coordinates in plane of plate } \\ a / b & - \text { aspect ratio } \\ M_{\zeta}, M_{\psi} & - \text { bending moment intensities in } \zeta \text { and } \psi \text { directions } \\ M_{\zeta \psi} & - \text { twisting moment intensity } \\ Y & - \text { Young's modulus } \\ D_{1} & - \text { flexural rigidity } \\ \phi(\zeta, \psi, t) & - \text { deflection of plate } \\ \Phi(\zeta, \psi) & - \text { deflection function } \\ \rho & - \text { mass density per unit volume of plate material } \\ t, T(t) & - \text { time and time function } \\ l & - \text { thickness of plate } \\ m & - \text { nonhomogeneity constant } \\ \alpha & - \text { thermal gradient } \\ \beta, \lambda & - \text { tapering and frequency parameter } \\ \nu & - \text { Poisson's ratio }\end{array}$

\section{Introduction}

The most of engineering structures such as rockets, missiles and nuclear reactors are working under sufficient temperature fields. Due to this temperature field, these structures undergo some vibrations. The excess of vibration causes system failure or its less efficiency. Therefore, scientists and engineers are very much keen to optimize the vibrations of structures. For this, they study the dynamic behavior of plates.

Amabili and Carra (2012) studied (experimentally) forced vibrations (large amplitude) of a thin rectangular plate (made of stainless-steel) carrying different concentrated masses. They 
tested the plate vertically and horizontally to analyze the gravity effect. They used an electrodynamic exciter and measured the vibration using a laser Doppler vibrometer. They showed in their results that concentrated masses do not effect in the vertical plate (trend of nonlinearity) but effect in the horizontal plate. A comprehensive study to analyze general vibration of rectangular orthotropic Kirchhoff plates using Trigonometry Ritz Method (TRM) was presented by Dozio (2011). The aim of this study was to extend the potential of the method on predicting natural flexural frequencies of plates. The author showed in his study that TRM had excellent accuracy when compared with other existing methods. Hota and Padhi (2007) applied the first order shear deformation theory to study vibration of amalgamation of a subparametric triangular plate. They analyzed free vibration of plates with cutouts as numerical examples. An analytical model to analyze vibration of partially cracked rectangular (isotropic) plates coupled with a fluid medium was proposed by Soni et al. (2018). They modified the governing equation of motion based on the classical plate theory (CPT) and evaluated fundamental frequencies. They also presented new results for fundamental frequencies affected by crack length, fluid level, fluid density and immersed depth of the plate.

Joshi et al. (2014) presented a model (analytically) to analyze nonlinear vibrations of cracked and isotropic rectangular plates. They used Galerkin's method to evaluate the natural frequencies of the plate with three different boundary conditions. They also showed that a crack parallel to the longer side of the plate affects vibration characteristics more as compared to the crack parallel to the shorter side. Ansari and Gupta (2003) discussed forced axisymmetric vibration of polar orthotropic circular plates with parabolic variation in thickness. They computed deflection and bending moments for the plate on different values of the taper, rigidity ratio and flexibility parameter. Bayat et al. (2011) analyzed nonlinear free vibrations (large amplitude) of tapered beams by using Max-Min Approach (MMA) and Homotopy Perturbation Method (HPM). They compared the results obtained by MMA and HPM with the exact solution to show the effectiveness of the method. The study of free vibration of a square plate was presented by Sharma et al. $(2016,2017)$. They showed the effect of circular variation in density and Poisson's ratio on vibrational frequency in two papers. In one paper, they showed that the frequency of vibration was less in the case of circular variation in density when compared to parabolic variation in density. In another paper, they showed that the rate of decrement in frequency modes was very less in the case of circular variation in Poisson's ratio.

Lam et al. (1989) presented the Rayleigh-Ritz method to study vibration of nonhomogeneous rectangular plates. They compared the obtained results (for isotropic and orthotropic plates) with the available results. Liew et al. (1995) provided a vast review of literature on vibration of thick plates. They focused mainly on the study of Mindlin theory, modified Mindlin plate theories for laminated plates and high order shear deformation plate theories. They categorized the study by different plate shapes. Lal et al. (2010) analyzed free transverse vibrations of uniform and nonhomogeneous rectangular plates using boundary characteristic orthogonal polynomials in the Rayleigh-Ritz method. They evaluated free vibration on four different combinations of clamped, simply supported and free edges. They studied linear variations in Young's modulus and density of the plate material and presented three dimensional mode shapes, for all the four boundary conditions. Free transverse vibrations of nonhomogeneous rectangular (orthotropic) plates resting on the Winkler foundation were presented by Kumar and Lal (2012) using twodimensional boundary characteristic orthogonal polynomials in the Rayleigh-Ritz method. They studied the effect of linear variation in density, two dimensional linear variations in thickness and the aspect ratio on the first three modes of vibration.

Khanna and Kaur (2014) presented an analysis of vibration of a tapered rectangular plate (nonhomogeneous) made up of a viscoelastic material. They studied the effect of linear variation in thickness, temperature and exponential variation in Poisson's ratio on the frequency of vibration. As a result, they showed that frequency modes are less in the case of linear variation in 
thickness when compared to exponential variation in thickness. They also showed that bilinear temperature variation provides less vibration as compared to exponential temperature variation. Chen et al. (2017) investigated transverse vibration of viscoelastic Timoshenko beam columns by using the generalized Hamilton principle. They showed the effect of length-to-depth ratio, the axial tension and viscosity coefficients on the natural frequencies and decrement coefficients. An asymmetric vibration of nonhomogeneous circular plates was investigated by Sharma et al. (2017). They analyzed the effect of parabolically varying thickness in the plate and exponential variation in Young's modulus as well as in density along the radial direction on the natural frequency.

In this study, the author finds the first two modes of vibration of a clamped rectangular plate corresponding to different values of nonhomogeneity, thermal gradient and the aspect ratio of the plate using the Rayleigh-Ritz method, based on the classical plate theory (CPT). Results are presented with the help of figures.

\section{Analysis}

The differential equation for transverse motion of the plate is

$$
\frac{\partial^{2} M_{\zeta}}{\partial \zeta^{2}}+2 \frac{\partial^{2} M_{\zeta \psi}}{\partial \zeta \partial \psi}+\frac{\partial^{2} M_{\psi}}{\partial \psi^{2}}=\rho l \frac{\partial^{2} \phi}{\partial t^{2}}
$$

where

$$
\begin{array}{ll}
M_{\zeta}=-D_{1}\left(\frac{\partial^{2} \phi}{\partial \zeta^{2}}+\nu \frac{\partial^{2} \phi}{\partial \psi^{2}}\right) & M_{\psi}=-D_{1}\left(\frac{\partial^{2} \phi}{\partial \psi^{2}}+\nu \frac{\partial^{2} \phi}{\partial \zeta^{2}}\right) \\
M_{\zeta \psi}=-D_{1}(1-\nu) \frac{\partial^{2} \phi}{\partial \zeta \partial \psi} &
\end{array}
$$

Substituting Eqs. (2.2) in Eq. (2.1), we get

$$
\begin{gathered}
{\left[D_{1}\left(\frac{\partial^{4} \phi}{\partial \zeta^{4}}+2 \frac{\partial^{4} \phi}{\partial \zeta^{2} \partial \psi^{2}}+\frac{\partial^{4} \phi}{\partial \psi^{4}}+\frac{\partial^{2} \nu}{\partial \zeta^{2}} \frac{\partial^{2} \phi}{\partial \psi^{2}}\right)+2 \frac{\partial D_{1}}{\partial \zeta}\left(\frac{\partial^{3} \phi}{\partial \zeta^{3}}+\frac{\partial^{3} \phi}{\partial \zeta \partial \psi^{2}}+\frac{\partial \nu}{\partial \zeta} \frac{\partial^{2} \phi}{\partial \psi^{2}}\right)\right.} \\
+2 \frac{\partial D_{1}}{\partial \psi}\left(\frac{\partial^{3} \phi}{\partial \psi^{3}}+\frac{\partial^{3} \phi}{\partial \psi \partial \zeta^{2}}-\frac{\partial \nu}{\partial \zeta} \frac{\partial^{2} \phi}{\partial \zeta \partial \psi}\right)+\frac{\partial^{2} D_{1}}{\partial \zeta^{2}}\left(\frac{\partial^{2} \phi}{\partial \zeta^{2}}+\nu \frac{\partial^{2} \phi}{\partial \psi^{2}}\right) \\
\left.+\frac{\partial^{2} D_{1}}{\partial \psi^{2}}\left(\frac{\partial^{2} \phi}{\partial \psi^{2}}+\nu \frac{\partial^{2} \phi}{\partial \zeta^{2}}\right)+2(1-\nu) \frac{\partial^{2} D_{1}}{\partial \zeta \partial \psi} \frac{\partial^{2} \phi}{\partial \zeta \partial \psi}\right]+\rho l \frac{\partial^{2} \phi}{\partial t^{2}}=0
\end{gathered}
$$

For the solution of Eq. (2.3), the deflection of the plate can be written as a product of the deflection and time function as

$$
\phi(\zeta, \psi, t)=\Phi(\zeta, \psi) T(t)
$$

Substituting Eq. (2.4) in Eq. (2.3), we get

$$
\begin{gathered}
T\left[D_{1}\left(\frac{\partial^{4} \Phi}{\partial \zeta^{4}}+2 \frac{\partial^{4} \Phi}{\partial \zeta^{2} \partial \psi^{2}}+\frac{\partial^{4} \Phi}{\partial \psi^{4}}+\frac{\partial^{2} \nu}{\partial \zeta^{2}} \frac{\partial^{2} \Phi}{\partial \psi^{2}}\right)+2 \frac{\partial D_{1}}{\partial \zeta}\left(\frac{\partial^{3} \Phi}{\partial \zeta^{3}}+\frac{\partial^{3} \Phi}{\partial \zeta \partial \psi^{2}}+\frac{\partial \nu}{\partial \zeta} \frac{\partial^{2} \Phi}{\partial \psi^{2}}\right)\right. \\
+2 \frac{\partial D_{1}}{\partial \psi}\left(\frac{\partial^{3} \Phi}{\partial \psi^{3}}+\frac{\partial^{3} \Phi}{\partial \psi \partial \zeta^{2}}-\frac{\partial \nu}{\partial \zeta} \frac{\partial^{2} \Phi}{\partial \zeta \partial \psi}\right)+\frac{\partial^{2} D_{1}}{\partial \zeta^{2}}\left(\frac{\partial^{2} \Phi}{\partial \zeta^{2}}+\nu \frac{\partial^{2} \Phi}{\partial \psi^{2}}\right) \\
\left.\quad+\frac{\partial^{2} D_{1}}{\partial \psi^{2}}\left(\frac{\partial^{2} \Phi}{\partial \psi^{2}}+\nu \frac{\partial^{2} \Phi}{\partial \zeta^{2}}\right)+2(1-\nu) \frac{\partial^{2} D_{1}}{\partial \zeta \partial \psi} \frac{\partial^{2} \Phi}{\partial \zeta \partial \psi}\right]+\rho l \Phi \frac{\partial^{2} T}{\partial t^{2}}=0
\end{gathered}
$$


Let us denote the expression in square brackets by $\mathcal{A}$. Now, by separating the variables, we get

$$
\frac{\mathcal{A}}{\rho l \Phi}=-\frac{1}{T} \frac{\partial^{2} T}{\partial t^{2}}=\omega^{2}
$$

where the expressions of Eq. (2.6) have been set equal to the constant $\omega^{2}$ because they are functions of independent variables $(\zeta, \psi)$ and $t$. Taking the first and last expression of Eq. (2.6), we have

$$
\mathcal{A}-\rho l \omega^{2} \Phi=0
$$

Equation (2.7) is a differential equation for transverse motion of the plate with the variable $D_{1}$ and $\nu$, i.e., the flexural rigidity and Poisson's ratio. The expression for flexural rigidity is given by $D_{1}=Y l^{3} /\left[12\left(1-\nu^{2}\right)\right]$.

\section{Construction of problem}

Consider a nonhomogeneous rectangular plate of length $a$ and breadth $b$ with one dimensional linear variation in thickness $l$ as shown in Fig. 1 is

$$
l=l_{0}\left(1+\beta \frac{\zeta}{a}\right)
$$

where $\beta(0 \leqslant \beta \leqslant 1)$ is known as the tapering parameter, and $l$ is constant at $\zeta=0$.

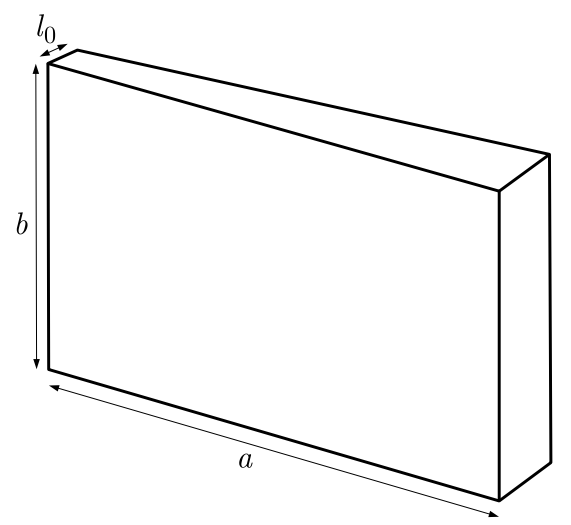

Fig. 1. Rectangular plate with linear variation in thickness

For consideration of a nonhomogeneous plate material, Poisson's ratio of the plate varies circular in one direction as (Kumar et al., 2018)

$$
\nu=\nu_{0}\left[1+m\left(1-\sqrt{1-\frac{\zeta^{2}}{a^{2}}}\right)\right]
$$

where $\nu_{0}$ is Poisson's ratio at $\zeta=0$ and $m(0 \leqslant m \leqslant 1)$ is known as the nonhomogeneity constant. The plate is subjected to steady two-dimensional temperature distributions as (Sharma et al., 2019)

$$
\tau=\tau_{0}\left(1-\frac{\zeta}{a}\right)\left(1-\frac{\psi}{b}\right)
$$


where $\tau$ and $\tau_{0}$ denote the temperature excess above the reference temperature on the plate at any point and at the origin, respectively. For most engineering materials, the temperature dependence of the modulus of elasticity is given by the relation

$$
Y=Y_{0}(1-\gamma \tau)
$$

where $Y_{0}$ is the Young's modulus at the mentioned temperature $\tau=0$ and $\gamma$ is called the slope of variation.

Using Eq. (3.3), Eq. (3.4) becomes

$$
Y=Y_{0}\left[1-\alpha\left(1-\frac{\zeta}{a}\right)\left(1-\frac{\psi}{b}\right)\right]
$$

where $\alpha(0 \leqslant \alpha<1)$ is called the temperature gradient, which is the product of temperature at the origin and slope of variation i.e., $\alpha=\gamma \tau_{0}$.

Using Eqs. (3.1), (3.2) and Eq. (3.5), the flexural rigidity of the plate becomes

$$
D_{1}=\frac{Y_{0} l_{0}^{3}\left[1-\alpha\left(1-\frac{\zeta}{a}\right)\left(1-\frac{\psi}{b}\right)\right]\left(1+\beta \frac{\zeta}{a}\right)^{3}}{12\left(1-\nu_{0}^{2}\left[1+m\left(1-\sqrt{1-\frac{\zeta^{2}}{a^{2}}}\right)\right]^{2}\right)}
$$

\section{Solution of the problem}

Author uses the Rayleigh-Ritz technique (i.e., maximum strain energy $V_{s}$ must be equal to maximum kinetic energy $T_{s}$ ) in order to obtain the frequency equation and frequencies for both modes of vibrations. Therefore, we must have

$$
\delta\left(V_{s}-T_{s}\right)=0
$$

Here, the expression for strain energy $V_{s}$ and kinetic energy $T_{s}$ is given by

$$
\begin{aligned}
& V_{s}=\frac{1}{2} \int_{0}^{a} \int_{0}^{b} D_{1}\left[\left(\frac{\partial^{2} \Phi}{\partial \zeta^{2}}\right)^{2}+\left(\frac{\partial^{2} \Phi}{\partial \psi^{2}}\right)^{2}+2 \nu \frac{\partial^{2} \Phi}{\partial \zeta^{2}} \frac{\partial^{2} \Phi}{\partial \psi^{2}}+2(1-\nu)\left(\frac{\partial^{2} \Phi}{\partial \zeta \partial \psi}\right)^{2}\right] d \psi d \zeta \\
& T_{s}=\frac{1}{2} \omega^{2} \rho \int_{0}^{a} \int_{0}^{b} l \Phi^{2} d \psi d \zeta
\end{aligned}
$$

Here, author is computing the frequency on the C-C-C-C condition (i.e., all edges of the plate are clamped). Therefore, the boundary condition for the clamped plate is given by

$$
\Phi=\frac{\partial \Phi}{\partial \zeta}=0 \quad \text { at } \quad \zeta=0, a \quad \Phi=\frac{\partial \Phi}{\partial \psi}=0 \quad \text { at } \quad \psi=0, b
$$

The deflection function which satisfies Eq. (4.3) is taken as

$$
\Phi(\zeta, \psi)=\left[\left(\frac{\zeta}{a}\right)^{2}\left(\frac{\psi}{b}\right)^{2}\left(1-\frac{\zeta}{a}\right)^{2}\left(1-\frac{\psi}{b}\right)^{2}\right]\left[\Omega_{1}+\Omega_{2}\left(\frac{\zeta}{a}\right)\left(\frac{\psi}{b}\right)\left(1-\frac{\zeta}{a}\right)\left(1-\frac{\psi}{b}\right)\right]
$$


where $\Omega_{1}$ and $\Omega_{2}$ are arbitrary constants. Substituting Eqs. (3.1), (3.2) and Eq. (3.6) into Eqs. (4.2), we get

$$
\begin{aligned}
V_{s}= & \frac{Y l_{0}^{3}}{24} \int_{0}^{a} \int_{0}^{b}\left[\frac{\left[1-\alpha\left(1-\frac{\zeta}{a}\right)\left(1-\frac{\psi}{b}\right)\right]\left(1+\beta \frac{\zeta}{a}\right)^{3}}{1-\nu_{0}^{2}\left[1+m\left(1-\sqrt{1-\frac{\zeta^{2}}{a^{2}}}\right)\right]^{2}}\right. \\
& \cdot\left\{\left(\frac{\partial^{2} \Phi}{\partial \zeta^{2}}\right)^{2}+\left(\frac{\partial^{2} \Phi}{\partial \psi^{2}}\right)^{2}+2 \nu_{0}\left[1+m\left(1-\sqrt{1-\frac{\zeta^{2}}{a^{2}}}\right)\right] \frac{\partial^{2} \Phi}{\partial \zeta^{2}} \frac{\partial^{2} \Phi}{\partial \psi^{2}}\right. \\
& \left.\left.+2\left(1-\nu_{0}\left[1+m\left(1-\sqrt{1-\frac{\zeta^{2}}{a^{2}}}\right)\right]\right)\left(\frac{\partial^{2} \Phi}{\partial \zeta \partial \psi}\right)^{2}\right\}\right] d \psi d \zeta
\end{aligned}
$$

and

$$
T_{s}=\frac{1}{2} \omega^{2} \rho l_{0} \int_{0}^{a} \int_{0}^{b}\left(1+\beta \frac{\zeta}{a}\right) \Phi^{2} d \psi d \zeta
$$

Now, convert $\zeta$ and $\psi$ into nondimensional variables as

$$
\zeta_{1}=\frac{\zeta}{a} \quad \psi_{1}=\frac{\psi}{a}
$$

Using Eq. (4.7), Eqs. (4.5) and (4.6) become

$$
\begin{aligned}
V_{s}^{*} & =\frac{Y l_{0}^{3}}{24 a^{2}} \int_{0}^{1} \int_{0}^{\frac{b}{a}}\left(\frac{\left[1-\alpha\left(1-\zeta_{1}\right)\left(1-\frac{a \psi_{1}}{b}\right)\right]\left(1+\beta \zeta_{1}\right)^{3}}{1-\nu_{0}^{2}\left[1+m\left(1-\sqrt{1-\zeta_{1}^{2}}\right)\right]^{2}}\right. \\
& \cdot\left\{\left(\frac{\partial^{2} \Phi}{\partial \zeta_{1}^{2}}\right)^{2}+\left(\frac{\partial^{2} \Phi}{\partial \psi_{1}^{2}}\right)^{2}+2 \nu_{0}\left[1+m\left(1-\sqrt{1-\zeta_{1}^{2}}\right)\right] \frac{\partial^{2} \Phi}{\partial \zeta_{1}^{2}} \frac{\partial^{2} \Phi}{\partial \psi_{1}^{2}}\right. \\
& \left.\left.+2\left(1-\nu_{0}\left[1+m\left(1-\sqrt{1-\zeta_{1}^{2}}\right)\right]\right)\left(\frac{\partial^{2} \Phi}{\partial \zeta_{1} \partial \psi_{1}}\right)^{2}\right\}\right) d \psi_{1} d \zeta_{1}
\end{aligned}
$$

and

$$
T_{s}^{*}=\frac{1}{2} \omega^{2} \rho l_{0} a^{2} \int_{0}^{1} \int_{0}^{\frac{b}{a}}\left(1+\beta \zeta_{1}\right) \Phi^{2} d \psi_{1} d \zeta_{1}
$$

Using Eqs. (4.8) and (4.9), Eq. (4.1) is converted into

$$
\delta\left(V_{s}^{*}-\lambda^{2} T_{s}^{*}\right)=0
$$

where $\lambda^{2}=12 \rho a^{4} \omega^{2} /\left(Y_{0} l_{0}^{2}\right)$ is a frequency parameter. Equation (4.10) consists of two unknown constants $\Omega_{1}$ and $\Omega_{2}$ (because of substitution of the deflection function $\Phi$ ). These two unknowns could be computed as follows

$$
\frac{\partial}{\partial \Omega_{n}}\left(V_{s}^{*}-\lambda^{2} T_{s}^{*}\right)=0 \quad n=1,2
$$


After simplifying Eq. (4.11), we get a homogeneous system of equations as

$$
f_{11} \Omega_{1}+f_{12} \Omega_{2}=0 \quad f_{21} \Omega_{1}+f_{22} \Omega_{2}=0
$$

In order to obtain a nontrivial or non-zero solution, the determinant of the coefficient matrix obtained from Eq. (4.12) must vanish. Therefore, we have

$$
\left|\begin{array}{ll}
f_{11} & f_{12} \\
f_{21} & f_{22}
\end{array}\right|=0
$$

Equation (4.13) is a quadratic equation from which we get two modes of vibration $\omega_{1}$ (first mode) and $\omega_{2}$ (second mode).

\section{Results and discussion}

In the calculations, the following parameters are used: $Y_{0}=7.08 \cdot 10^{10} \mathrm{n} / \mathrm{m}^{2}, \nu_{0}=0.345$, $\rho=2.80 \cdot 10^{3} \mathrm{~kg} / \mathrm{m}^{3}, l_{0}=0.01 \mathrm{~m}$.

Figure 2 shows the vibrational frequency corresponding to the nonhomogeneity constant $m$ for a fixed value of the aspect ratio $a / b=1.5$ and for the following combination of the tapering parameter $\beta$ and thermal gradient $\alpha(\beta=\alpha=0.0, \beta=\alpha=0.6)$.
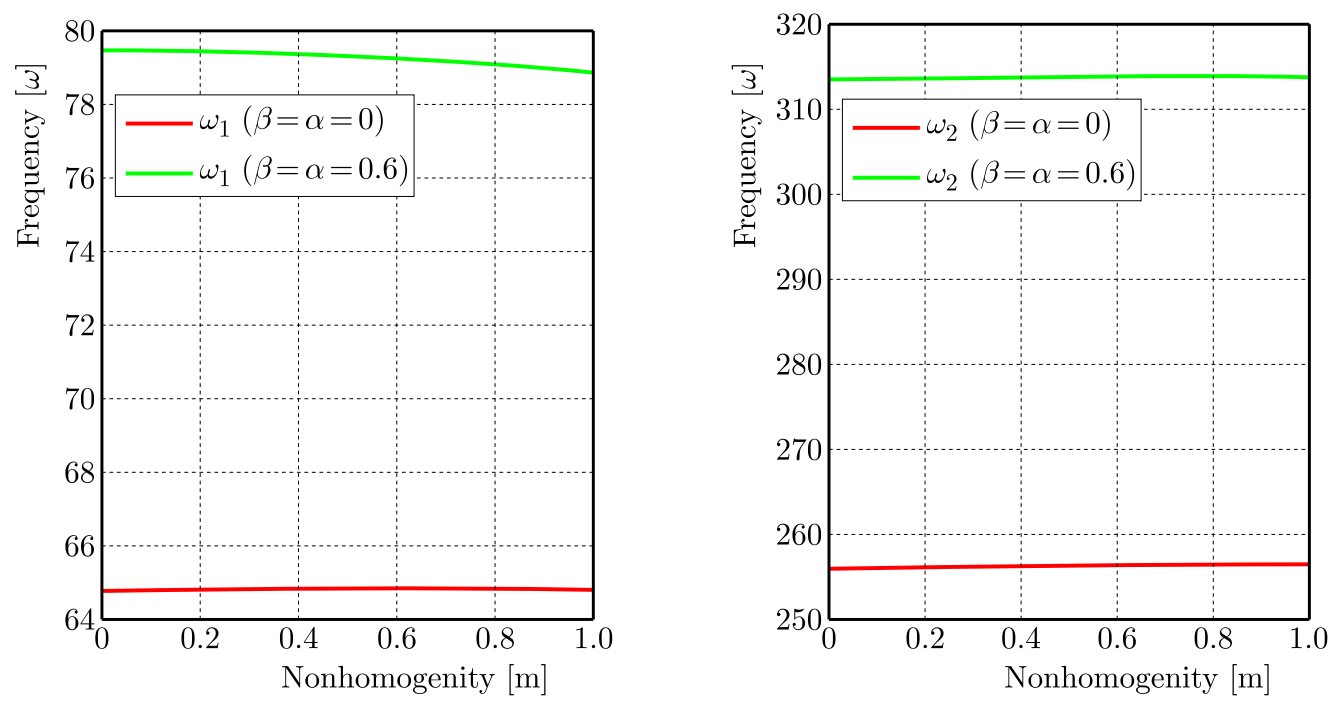

Fig. 2. Vibrational frequency $\omega$ corresponding to nonhomogeneity $m$

The frequency of mode of vibration increases with the increasing value of the nonhomogeneity constant $m$ for both the combination of the tapering constant $\beta$ and thermal gradient $\alpha$. The rate of the increment is very small (almost constant) due to circular variation in nonhomogeneity. When $\beta=\alpha=0.6$, the first mode frequency is slightly decreasing as shown in Fig. 2 . The frequency also increases when the combined value of the tapering constant $\beta$ and thermal gradient $\alpha$ varies from 0.0 to 0.6 .

The frequency of vibration corresponding to the thermal gradient $\alpha$ for a fixed value of the aspect ratio $a / b=1.5$ is presented in Fig. 3 for the following combination of the nonhomogeneity constant $m$ and the tapering constant $\beta(\beta=m=0.0, \beta=m=0.6)$.

The frequency of both modes decreases when temperature variation in the plate increases from 0.0 to 0.8 . It is also noted that when the combined value of the tapering constant $\beta$ and nonhomogeneity constant $m$ increases from 0.0 to 0.6 , the frequency increases. 

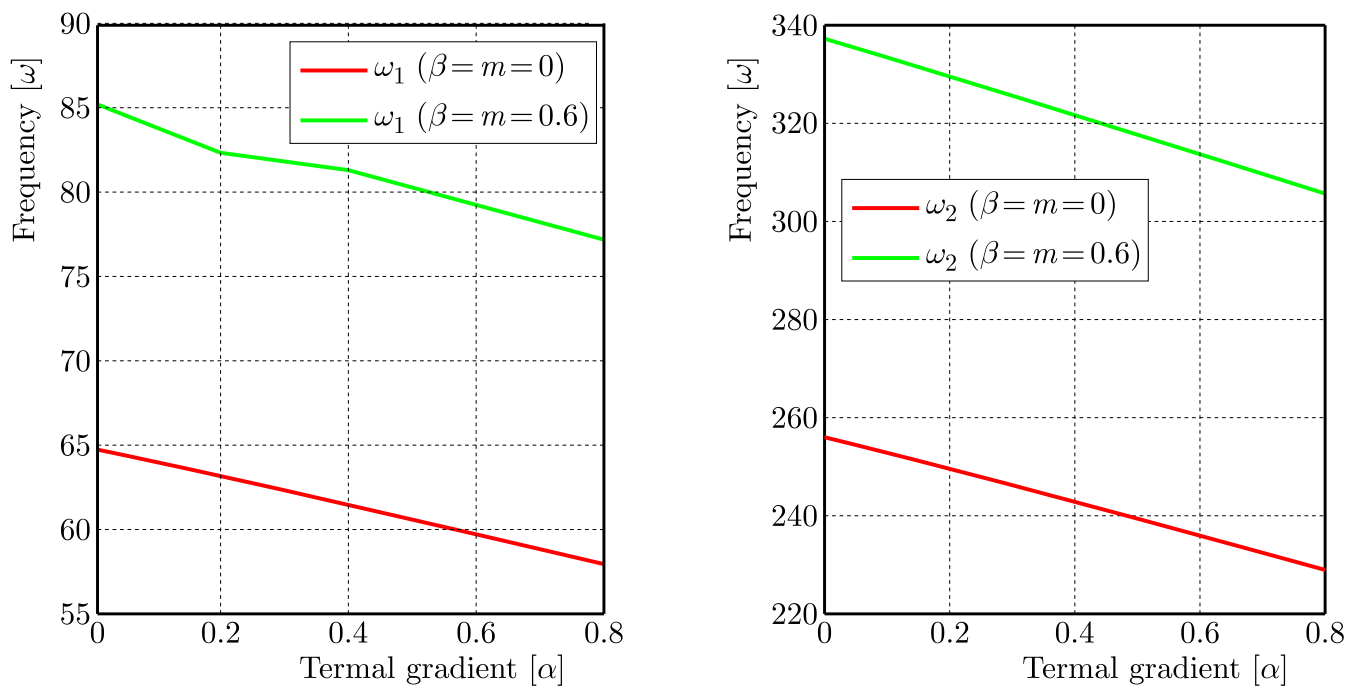

Fig. 3. Vibrational frequency $\omega$ corresponding to thermal gradient $\alpha$

Figure 4 provides the vibrational frequency for different values of the aspect ratio $a / b$ for the following combination of the tapering constant $\beta$, thermal gradient $\alpha$ and the nonhomogeneity constant $m(\beta=\alpha=m=0.2, \beta=\alpha=m=0.4)$.
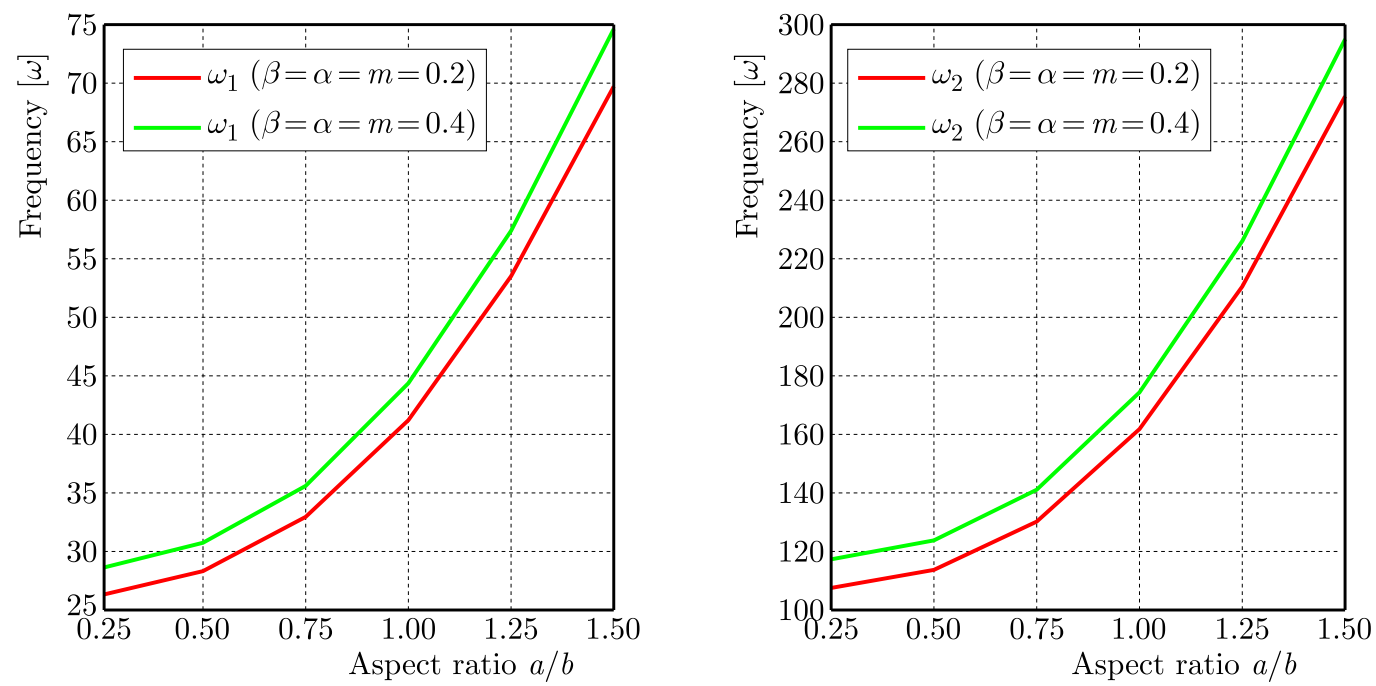

Fig. 4. Vibrational frequency $\omega$ corresponding to aspect ratio $a / b$

From Fig. 4, it is noticed that frequency of both modes of vibration increases rapidly with an increasing value of the aspect ratio $a / b$. Also a very small increment is noted in both modes when the combined value of the tapering constant $\beta$, thermal gradient $\alpha$ and the nonhomogeneity constant $m$ increases from 0.2 to 0.4

\section{Comparison of results}

A comparison of the frequency of modes from the present paper with those obtained by Khanna and Kaur (2014) is presented in Tables 1 and 2.

Table 1 provides the comparison of the frequency of modes with Khanna and Kaur (2014) corresponding to the nonhomogeneity constant $m$ for the following cases: 1) $\beta=\alpha=0.0$, 2) $\beta=\alpha=0.2,3) \beta=\alpha=0.6$. 
Table 1. Comparison of the mode frequencies of the present paper with Khanna and Kaur (2014) corresponding to the nonhomogeneity constant $m$

\begin{tabular}{|c|c|c|c|c|c|c|}
\hline \multirow{2}{*}{$m$} & \multicolumn{2}{|c|}{$\beta=\alpha=0.0$} & \multicolumn{2}{c|}{$\beta=\alpha=0.2$} & \multicolumn{2}{c|}{$\beta=\alpha=0.6$} \\
\cline { 2 - 7 } & $\omega_{1}$ & $\omega_{2}$ & $\omega_{1}$ & $\omega_{2}$ & $\omega_{1}$ & $\omega_{2}$ \\
\hline \hline \multirow{2}{*}{0.00} & 64.77 & 255.98 & 69.67 & 275.29 & 79.47 & 313.50 \\
& $\mathbf{6 4 . 7 7}$ & $\mathbf{2 5 5 . 9 8}$ & $\mathbf{6 9 . 6 7}$ & $\mathbf{2 7 5 . 2 9}$ & $\mathbf{7 9 . 4 7}$ & $\mathbf{3 1 3 . 5 0}$ \\
\hline \multirow{2}{*}{0.05} & 64.78 & 256.02 & 69.68 & 275.32 & 79.46 & 313.55 \\
& $\mathbf{6 4 . 9 9}$ & $\mathbf{2 5 6 . 8 4}$ & $\mathbf{6 9 . 9 1}$ & $\mathbf{2 7 6 . 2 5}$ & $\mathbf{7 9 . 7 5}$ & $\mathbf{3 1 4 . 6 8}$ \\
\hline \multirow{2}{*}{0.10} & 64.79 & 256.05 & 69.68 & 275.36 & 79.45 & 313.58 \\
& $\mathbf{6 5 . 2 2}$ & $\mathbf{2 5 7 . 7 6}$ & $\mathbf{7 0 . 1 7}$ & $\mathbf{2 7 7 . 2 9}$ & $\mathbf{8 0 . 0 5}$ & $\mathbf{3 1 5 . 9 3}$ \\
\hline \multirow{2}{*}{0.15} & 64.80 & 256.08 & 69.69 & 275.40 & 79.44 & 313.62 \\
& $\mathbf{6 5 . 4 7}$ & $\mathbf{2 5 8 . 7 4}$ & $\mathbf{7 0 . 4 5}$ & $\mathbf{2 7 8 . 3 9}$ & $\mathbf{8 0 . 3 8}$ & $\mathbf{3 1 7 . 2 9}$ \\
\hline
\end{tabular}

The values written in bold are from Khanna and Kaur (2014)

Table 1 provides the information that the frequency of modes in the present paper are less when compared to the frequency obtained by Khanna and Kaur (2014) for all the three cases. The frequencies of the present study are equal to mode frequencies obtained by Khanna and Kaur (2014) at $\alpha=\beta=m=0.0$.

Tables 2 shows the comparison of the frequency of modes with Khanna and Kaur (2014) corresponding to the aspect ratio $a / b$ for the following cases: 4$) \alpha=\beta=m=0.0,5) \alpha=\beta=0.2$, $m=0.1$.

Table 2. Comparison of the mode frequencies of the present paper with Khanna and Kaur (2014) corresponding to the aspect ratio $a / b$

\begin{tabular}{|c|c|c|c|c|}
\hline \multirow{2}{*}{$a / b$} & \multicolumn{2}{|c|}{$\alpha=\beta=m=0$} & \multicolumn{2}{c|}{$\alpha=\beta=0.2, m=0.1$} \\
\cline { 2 - 5 } & $\omega_{1}$ & $\omega_{2}$ & $\omega_{1}$ & $\omega_{2}$ \\
\hline \hline \multirow{2}{*}{0.25} & 24.30 & 99.00 & 26.32 & 108.13 \\
& $\mathbf{2 4 . 3 0}$ & $\mathbf{9 9 . 0 0}$ & $\mathbf{2 6 . 5 1}$ & $\mathbf{1 0 8 . 1 3}$ \\
\hline \multirow{2}{*}{0.50} & 26.22 & 104.86 & 28.37 & 113.58 \\
& $\mathbf{2 6 . 2 2}$ & $\mathbf{1 0 4 . 8 6}$ & $\mathbf{2 8 . 5 8}$ & $\mathbf{1 1 4 . 4 1}$ \\
\hline \multirow{2}{*}{0.75} & 30.58 & 120.33 & 33.04 & 130.09 \\
& $\mathbf{3 0 . 5 8}$ & $\mathbf{1 2 0 . 3 3}$ & $\mathbf{3 3 . 2 8}$ & $\mathbf{1 3 1 . 0 3}$ \\
\hline \multirow{2}{*}{1.00} & 38.32 & 149.97 & 41.33 & 161.77 \\
& $\mathbf{3 8 . 3 2}$ & $\mathbf{1 4 9 . 9 7}$ & $\mathbf{4 1 . 6 2}$ & $\mathbf{1 6 2 . 9 2}$ \\
\hline \multirow{2}{*}{1.25} & 49.75 & 195.35 & 53.57 & 210.37 \\
& $\mathbf{4 9 . 7 5}$ & $\mathbf{1 9 5 . 3 5}$ & $\mathbf{5 3 . 9 5}$ & $\mathbf{2 1 1 . 8 5}$ \\
\hline \multirow{2}{*}{1.50} & 64.77 & 255.98 & 69.68 & 275.36 \\
& $\mathbf{7 4 . 7 7}$ & $\mathbf{2 5 5 . 9 8}$ & $\mathbf{7 0 . 1 7}$ & $\mathbf{2 7 7 . 2 9}$ \\
\hline
\end{tabular}

The values written in bold are from Khanna and Kaur (2014)

It is noticed from the comparison that mode frequencies of the present paper are the same as those obtained by Khanna and Kaur (2014) for case 4. But for case 5, the mode frequencies of the present paper are less when compared to those obtained by Khanna and Kaur (2014).

\section{Conclusion}

In this paper, the author attempts to present a mathematical model to analyze vibration of a nonuniform and nonhomogeneous rectangular plate under a temperature field. From the results and discussion and comparison, the author concludes as in the following: 
- The mode frequencies are less in the case of circular variation in nonhomogeneity (present paper) when compared to exponential variation in nonhomogeneity (Khanna and Kaur, 2014) as shown in Table 1. The mode frequencies are the same with the frequencies obtained by Khanna and Kaur (2014) when $\alpha=\beta=m=0.0$.

- The mode frequencies are same with frequencies obtained by Khanna and Kaur (2014) corresponding to the aspect ratio $a / b$ when the nonhomogeneity constant is kept zero i.e., $m=0$. But when the nonhomogeneity is not zero $(m=0.1)$ then the frequencies of the present paper are less in comparison with the modes obtained by Khanna and Kaur (2014) as shown in Table 2.

- The variation in the frequencies are less due to circular variation in nonhomogeneity as shown in Fig. 2.

- The vibration frequency decreases due to bilinear temperature variation in the plate as shown in Fig. 3.

\section{References}

1. Ansari A.H., Gupta U.S., 2003, Transverse vibration of polar orthotropic parabolically tapered circular plate, Indian Journal of Pure and Applied Mathematics, 34, 6, 819-830

2. Amabili M., Carra S., 2012, Experiments and simulations for large-amplitude vibrations of rectangular plates carrying concentrated masses, Journal of Sound and Vibration, 331, 155-166

3. Bayat M., Pakar I., Bayat M., 2011, Analytical study on the vibration frequencies of tapered beams, Latin American Journal of Solids and Structures, 8, 149-162

4. Chen L.Q., Peng L., Zhan A.Q., 2017, Transverse vibration of viscoelastic Timoshenko beam columns, Journal of Vibration and Control, 23, 10, 1572-1584

5. Dozio L., 2011, On the use of the trigonometric Ritz method for general vibration analysis of rectangular Kirchhoff plates, Thin-Walled Structures, 49, 129-144

6. Hota S.S., PAdhi P., 2007, Vibration of plates with arbitrary shapes of cutouts, Journal of Sound and Vibration, 302, 1030-1036

7. Joshi P.V., Jain N.K., RamtekKar G.D., 2014, Analytical modeling and vibration analysis of internally cracked rectangular plates, Journal of Sound and Vibration, 333, 5851-5864

8. Khanna A., KaUR N., 2014, A study on vibration of tapered rectangular plate under non uniform temperature field, Mechanics, 20, 4, 376-381

9. Kumar A., Lather N., Bhardwaj R., Mani N., Sharma A., 2018, Effect of linear variation in density and circular variation in Poisson's ratio on time period of vibration of rectangular plate, Vibroengineering Procedia, 21, 14-19

10. Kumar Y., LaL R., 2012, Vibrations of nonhomogeneous orthotropic rectangular plates with bilinear thickness variation resting on Winkler foundation, Meccanica, 47, 893-915

11. Lal R., Kumar Y., Gupta U.S., 2010, Transverse vibrations of nonhomogeneous rectangular plates of uniform thickness using boundary characteristic orthogonal polynomials, International Journal of Applied Mathematics and Mechanics, 6, 14, 93-109

12. Lam K.Y., Hung K.C., Chow S.T., 1989, Vibration analysis of plates with cutouts by the modified Rayleigh-Ritz method, Applied Acoustics, 28, 49-60

13. Liew K.M., Xiang Y., Kitipornchai S., 1995, Research on thick plate vibration: A literature survey, Journal of Sound and Vibration, 180, 163-176

14. Sharma A., Sharma A.K., Raghav A.K., Kumar V., 2016, Vibrational study of square plate with thermal effect and circular variation in density, Romanian Journal of Acoustics and Vibration, 13, $146-152$ 
15. Sharma A., Kumar V., Raghav A.K., 2017, Vibrational frequency of circular tapered square plate, Romanian Journal of Acoustics and Vibration, 14, 21-27

16. Sharma A., Mani N., Bhardwaj R., 2019, Natural vibration of tapered rectangular plate with exponential variation in non homogeneity, Journal of Vibroengineering, 21, 187-197

17. Sharma S., Lal R., Singh N., 2017, Effect of non-homogeneity on asymmetric vibrations of non-uniform circular plates, Journal of Vibration and Control, 23, 10, 1635-1644

18. Soni S., Jain N.K., Joshi P.V., 2018, Vibration analysis of partially cracked plate submerged in fluid, Journal of Sound and Vibration, 412, 28-57

Manuscript received February 7, 2018; accepted for print March 1, 2019 